Commun. Fac. Sci. Univ. Ank. Ser. A1 Math. Stat.

Volume 68, Number 2, Pages 1774-1779(2019)

DOI: $10.31801 /$ cfsuasmas. 457500

ISSN 1303-5991 E-ISSN 2618-6470

http://communications.science.ankara.edu.tr/index.php?series=A1

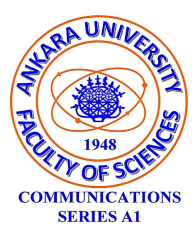

\title{
THE DIMENSION OF PRODUCTS OF $n$ HOMOGENEOUS COMPONENTS IN FREE LIE ALGEBRAS
}

\author{
NIL MANSUROGLU
}

\begin{abstract}
Let $L$ be a free Lie algebra of finite rank $r \geq 2$ over a field $F$ and we let $L_{m_{i}}$ denote the degree $m_{i}$ homogeneous component of $L$. Ralph Stöhr and Micheal Vaughan-Lee derived formulae for the dimension of the subspaces $\left[L_{m_{1}}, L_{m_{2}}\right]$ for all $m_{1}$ and $m_{2}$. Then, the author and R. Stöhr obtained formulae for the dimension of the products $\left[L_{m_{1}}, L_{m_{2}}, L_{m_{3}}\right]$ under certain conditions on $m_{1}, m_{2}, m_{3}$. In this paper, we study on products of $n$ homogeneous components in free Lie algebra and we derive formulae for the dimension of such products.
\end{abstract}

\section{INTRODUCTION}

Let $L$ be a free Lie algebra of finite rank $r$ over a field $F$ and let $L_{m_{i}}$ denote the degree $m_{i}$ homogeneous component of $L$. The algebra $L$ has a natural graded as

$$
L=L_{1} \oplus L_{2} \oplus \ldots \oplus L_{m_{i}} \oplus \ldots
$$

Throughout of this paper, we use the left normed convention for Lie brackets, that is, for $a_{1}, \ldots, a_{s} \in L$ we write $\left[a_{1}, a_{2}, \ldots, a_{s}\right]=\left[\left[a_{1}, a_{2}, \ldots, a_{s-1}\right], a_{s}\right]$. For calculating the dimension of $L_{m_{i}}$, we use Witt's formula

$$
\operatorname{dim} L_{m_{i}}=f\left(m_{i}, r\right)=\frac{1}{m_{i}} \sum_{d \mid m_{i}} \mu(d) r^{\frac{m_{i}}{d}},
$$

where $\mu$ is the Möbius function (see [6, 7, [1, Theorem 5.11]). Moreover, in this note, since $\left[L_{m_{1}}, L_{m_{2}}\right]=\left[L_{m_{2}}, L_{m_{1}}\right]$ for all $m_{1}$ and $m_{2}$, we focus on the case $m_{1} \geq m_{2}$.

Take a subset $Y$ of $L$, let $L(Y)$ be the Lie subalgebra generated by $Y$ in $L$ and we denote the degree $m_{i}$ homogeneous component of $L(Y)$ by $L_{m_{i}}(Y)$. We

Received by the editors: September 05, 2018, Accepted: December 29, 2018.

2010 Mathematics Subject Classification. 17B01.

Key words and phrases. Free Lie algebras, homogeneous component.

This work was supported by Ahi Evran University Scientific Research Projects Coordination Unit. Project Number: FEF.A4.18.009. 
say that a set $Y$ of homogeneous elements in $L$ is a reduced set if none of its elements is contained in the subalgebra of $L$ generated by the remaining elements of $Y$.

The following lemma is referred to as Shirshov's Lemma which is played an important role in the proof of the Shirshov-Witt Theorem (see [3, 4]).

Lemma 1 (3), Proof of Theorem 2). If $L$ is a free Lie algebra and $Y$ is a reduced set of homogeneous elements in $L$, then $Y$ is a set of free generators for the subalgebra $L(Y)$.

Given any homogeneous subspace $U$ in $L_{m_{i}}$. The Lie subalgebra $L(U)$ is free of rank $\operatorname{dim} U$ and any $F$-basis of $U$ is a free generating set for the Lie subalgebra $L(U)$. In [2, the author and R. Stöhr investigated the dimension of product of two homogeneous subspaces and they proved the following lemma which will be the key step in the proof of our main results.

Lemma 2 ([2], Lemma 2.2). Let $U$ and $V$ be subspaces of $L$ such that $U \subseteq L_{m_{1}}, V \subseteq$ $L_{m_{2}}$ with $m_{1} \geq m_{2} \geq 1$. Then

$$
\operatorname{dim}[U, V]=\operatorname{dim}[U \cap L(V), V]+(\operatorname{dim} U-\operatorname{dim}(U \cap L(V))) \operatorname{dim} V .
$$

\section{Products of $n$ Homogeneous components}

In this paper, our aim is to investigate the dimension of the subspaces in the forms $\left[L_{m_{1}}, L_{m_{2}}, \ldots, L_{m_{n}}\right]$. Firstly, we give a technical lemma for the proof of main result.

Lemma 3. Let $m_{1}, m_{2}, \ldots, m_{n}$ be positive integers with $m_{1} \geq m_{2}$.

(i) If $m_{1}=s_{1} m_{n}, m_{2}=s_{2} m_{n}, \ldots, m_{n-1}=s_{n-1} m_{n}$ for some positive integers $s_{1}, s_{2}, \ldots, s_{n-1}$, then

$$
\left[L_{m_{1}}, L_{m_{2}}, \ldots, L_{m_{n-1}}\right] \cap L\left(L_{m_{n}}\right)=\left[L_{s_{1}}\left(L_{m_{n}}\right), L_{s_{2}}\left(L_{m_{n}}\right), \ldots, L_{s_{n-1}}\left(L_{m_{n}}\right)\right] \text {. }
$$

(ii) If at least one of $m_{1}, m_{2}, \ldots, m_{n-1}$ is not divided by $m_{n}$, then

$$
\left[L_{m_{1}}, L_{m_{2}}, \ldots, L_{m_{n-1}}\right] \cap L\left(L_{m_{n}}\right)=0 .
$$

Proof. (i) Clearly, we have

$$
\left[L_{s_{1}}\left(L_{m_{n}}\right), L_{s_{2}}\left(L_{m_{n}}\right), \ldots, L_{s_{n-1}}\left(L_{m_{n}}\right)\right] \subseteq\left[L_{m_{1}}, L_{m_{2}}, \ldots, L_{m_{n-1}}\right] \cap L\left(L_{m_{n}}\right) .
$$

It remains to show that

$$
\left[L_{m_{1}}, L_{m_{2}}, \ldots, L_{m_{n-1}}\right] \cap L\left(L_{m_{n}}\right) \subseteq\left[L_{s_{1}}\left(L_{m_{n}}\right), L_{s_{2}}\left(L_{m_{n}}\right), \ldots, L_{s_{n-1}}\left(L_{m_{n}}\right)\right] .
$$

Firstly, we take the subalgebra $L^{m_{n}}=L_{m_{n}} \oplus L_{m_{n}+1} \oplus L_{m_{n}+2} \oplus \ldots$, this is the $m_{n}$-th term of the lower central series of $L$. This subalgebra has a homogeneous free generating set of the form $\mathcal{S}=\mathcal{S}_{m_{n}} \cup \mathcal{S}_{m_{n}+1} \cup \mathcal{S}_{m_{n}+2} \cup \ldots$, where $\mathcal{S}_{i} \subset L_{i}$ $\left(i=m_{n}, m_{n}+1, \ldots\right)$. It is easy to obtain a free generating set for $L^{m_{n}}$. First, we consider an $F$-basis of $L_{m_{n}}$ for $\mathcal{S}_{m_{n}}$, then we proceed inductively by taking a basis of a vector space complement of $L_{i} \cap L\left(\mathcal{S}_{m_{n}} \cup \ldots \cup \mathcal{S}_{i-1}\right)$ as the set $\mathcal{S}_{i}$ for $i>m_{n}$. 
By Lemma 1, it is clear to verify that the set is a generating set for $L^{m_{n}}$ and also it is a free generating set. Hence, $L_{m_{n}}=\left\langle\mathcal{S}_{m_{n}}\right\rangle$ and $\mathcal{S}_{m_{n}}$ is a free generating set for $L\left(L_{m_{n}}\right)$.

We now consider a projection map $\pi: L^{m_{n}} \rightarrow L\left(L^{m_{n}}\right)$ defined by $\pi(x)=$ $x$ for $x \in \mathcal{S}_{m_{n}}$ and $\pi(x)=0$ for $x \in \mathcal{S} \backslash \mathcal{S}_{m_{n}}$. This map is also a Lie algebra homomorphism. Arbitrary chosen an element $\mathcal{B}$ in $\left[L_{m_{1}}, L_{m_{2}}, \ldots, L_{m_{n-1}}\right]$ can be expressed as a linear combination of the Lie products $\left[u_{1_{j}}, u_{2 j}, \ldots, u_{(n-1)}{ }_{j}\right.$ for $u_{1 j} \in L_{m_{1}}, u_{2 j} \in L_{m_{2}}, \ldots, u_{(n-1)} \in L_{m_{n-1}}$, namely,

$$
\mathcal{B}=\sum_{j} \alpha_{j}\left[u_{1 j}, u_{2 j}, \ldots, u_{(n-1)}\right]
$$

for some scalars $\alpha_{j} \in F$. Here, $\pi\left(u_{1 j}\right) \in L_{s_{1}}\left(L_{m_{n}}\right), \pi\left(u_{2 j}\right) \in L_{s_{2}}\left(L_{m_{n}}\right), \ldots, \pi\left(u_{(n-1)_{j}}\right) \in$ $L_{s_{n-1}}\left(L_{m_{n}}\right)$. Suppose that $\mathcal{B} \in L\left(L_{m_{n}}\right)$. Since $\pi$ is a Lie algebra homomorphism, we have

$$
\begin{aligned}
& \mathcal{B}=\pi(\mathcal{B})=\pi\left(\sum_{j} \alpha_{j}\left[u_{1 j}, u_{2 j}, \ldots, u_{(n-1)}\right]\right) \\
& =\sum_{j} \alpha_{j}\left[\pi\left(u_{1 j}\right), \pi\left(u_{2 j}\right), \ldots, \pi\left(u_{(n-1)}\right)\right] \in\left[L_{s_{1}}\left(L_{m_{n}}\right), L_{s_{2}}\left(L_{m_{n}}\right), \ldots, L_{s_{n-1}}\left(L_{m_{n}}\right)\right] .
\end{aligned}
$$

Therefore, we proved the inverse inclusion

$$
\left[L_{m_{1}}, L_{m_{2}}, \ldots, L_{m_{n-1}}\right] \cap L\left(L_{m_{n}}\right) \subseteq\left[L_{s_{1}}\left(L_{m_{n}}\right), L_{s_{2}}\left(L_{m_{n}}\right), \ldots, L_{s_{n-1}}\left(L_{m_{n}}\right)\right] .
$$

(ii) Here in order to prove this part of lemma, we use the projection $\pi$ as in (i) and we choose arbitrary element in $L\left(L_{m_{n}}\right)$, say $\mathcal{B}=\sum_{j} \alpha_{j}\left[u_{1 j}, u_{2 j}, \ldots, u_{(n-1)_{j}}\right]$ with $u_{1 j} \in L_{m_{1}}, u_{2 j} \in L_{m_{2}}, \ldots, u_{(n-1)_{j}} \in L_{m_{n-1}}$. Since any element in $L\left(L_{m_{n}}\right)$ is written as a linear combination of elements of degree $\lambda m_{n}$ with $\lambda=1,2, \ldots$, all homogeneous components $L_{s}$ with $s \geq m_{n}$ and $m_{n} \nmid s$ are in the kernel of $\pi$. As $m_{n} \nmid$ $m_{1}$ or $m_{n} \nmid m_{2}$ or $\ldots$ or $m_{n} \nmid m_{n-1}$, at least one of $\pi\left(u_{1 j}\right), \pi\left(u_{2 j}\right), \ldots, \pi\left(u_{(n-1)}\right)$ ) is zero. Thus,

$$
\begin{aligned}
\pi\left(\left[u_{1 j}, u_{2 j}, \ldots, u_{(n-1)}\right]\right) & =\left[\pi\left(u_{1 j}\right), \pi\left(u_{2 j}\right), \ldots, \pi\left(u_{(n-1)}\right)\right] \\
& =0
\end{aligned}
$$

for all $j$. Therefore, we conclude that

$$
\left[L_{m_{1}}, L_{m_{2}}, \ldots, L_{m_{n-1}}\right] \cap L\left(L_{m_{n}}\right)=0
$$

as required.

We are now ready to give main theorem.

Theorem 4. Let $m_{1}, m_{2}, \ldots, m_{n}$ be positive integers with $m_{1} \geq m_{2}$.

(i) If $m_{1}+m_{2}+\ldots+m_{n-1}>m_{n}$ and at least one of $m_{1}, m_{2}, \ldots, m_{n-1}$ is not divided by $m_{n}$, then

$$
\operatorname{dim}\left[L_{m_{1}}, L_{m_{2}}, \ldots, L_{m_{n}}\right]=\operatorname{dim}\left[L_{m_{1}}, L_{m_{2}}, \ldots, L_{m_{n-1}}\right] \operatorname{dim} L_{m_{n}},
$$


(ii) if $m_{1}+m_{2}+\ldots+m_{n-1}>m_{n}$ and $m_{1}=s_{1} m_{n}, m_{2}=s_{2} m_{n}, \ldots, m_{n-1}=s_{n-1} m_{n}$ for some positive integers $s_{1}, s_{2}, \ldots, s_{n-1}$, then

$$
\operatorname{dim}\left[L_{m_{1}}, L_{m_{2}}, \ldots, L_{m_{n}}\right]=\operatorname{dim}\left[L_{s_{1}}\left(L_{m_{n}}\right), L_{s_{2}}\left(L_{m_{n}}\right), \ldots, L_{s_{n-1}}\left(L_{m_{n}}\right), L_{m_{n}}\right]
$$

$+\left(\operatorname{dim}\left[L_{m_{1}}, L_{m_{2}}, \ldots, L_{m_{n-1}}\right]-\operatorname{dim}\left[L_{s_{1}}\left(L_{m_{n}}\right), L_{s_{2}}\left(L_{m_{n}}\right), \ldots, L_{s_{n-1}}\left(L_{m_{n}}\right)\right]\right) \operatorname{dim} L_{m_{n}}$,

(iii) if $m_{n} \geq m_{1}+m_{2}+\ldots+m_{n-1}$ and $\left(m_{1}+m_{2}+\ldots+m_{n-1}\right) \nmid m_{n}$, then

$$
\operatorname{dim}\left[L_{m_{1}}, L_{m_{2}}, \ldots, L_{m_{n}}\right]=\operatorname{dim}\left[L_{m_{1}}, L_{m_{2}}, \ldots, L_{m_{n-1}}\right] \operatorname{dim} L_{m_{n}},
$$

(iv) and if $m_{n} \geq m_{1}+m_{2}+\ldots+m_{n-1}$ and $m_{n}=s\left(m_{1}+m_{2}+\ldots+m_{n-1}\right)$ with $s \geq 1$, then

$$
\begin{array}{r}
\operatorname{dim}\left[L_{m_{1}}, L_{m_{2}}, \ldots, L_{m_{n}}\right]=\operatorname{dim} L_{s+1}\left(\left[L_{m_{1}}, L_{m_{2}}, \ldots, L_{m_{n-1}}\right]\right) \\
+\left(\operatorname{dim} L_{m_{n}}-\operatorname{dim} L_{s}\left(\left[L_{m_{1}}, L_{m_{2}}, \ldots, L_{m_{n-1}}\right]\right)\right) \operatorname{dim}\left[L_{m_{1}}, L_{m_{2}}, \ldots, L_{m_{n-1}}\right] .
\end{array}
$$

Proof. (i) We apply Lemma 2 with $U=\left[L_{m_{1}}, L_{m_{2}}, \ldots, L_{m_{n-1}}\right]$ and $V=L_{m_{n}}$. Thus we get

$$
\begin{array}{r}
\operatorname{dim}\left[L_{m_{1}}, L_{m_{2}}, \ldots, L_{m_{n}}\right]=\operatorname{dim}\left[\left[L_{m_{1}}, L_{m_{2}}, \ldots, L_{m_{n-1}}\right] \cap L\left(L_{m_{n}}\right), L_{m_{n}}\right] \\
+\left(\operatorname{dim}\left[L_{m_{1}}, L_{m_{2}}, \ldots, L_{m_{n-1}}\right]-\operatorname{dim}\left(\left[L_{m_{1}}, L_{m_{2}}, \ldots, L_{m_{n-1}}\right] \cap L\left(L_{m_{n}}\right)\right)\right) \operatorname{dim} L_{m_{n}} .
\end{array}
$$

By Lemma 3 (ii), we have $\left[L_{m_{1}}, L_{m_{2}}, \ldots, L_{m_{n-1}}\right] \cap L\left(L_{m_{n}}\right)=0$. This gives

$$
\operatorname{dim}\left[L_{m_{1}}, L_{m_{2}}, \ldots, L_{m_{n-1}}\right]=\operatorname{dim}\left[L_{m_{1}}, L_{m_{2}}, \ldots, L_{m_{n-1}}\right] \operatorname{dim} L_{m_{n}} .
$$

(ii) By applying Lemma 2 with $U=\left[L_{m_{1}}, L_{m_{2}}, \ldots, L_{m_{n-1}}\right]$ and $V=L_{m_{n}}$, we get

$$
\begin{array}{r}
\operatorname{dim}\left[L_{m_{1}}, L_{m_{2}}, \ldots, L_{m_{n}}\right]=\operatorname{dim}\left[\left[L_{s_{1} m_{n}}, L_{s_{2} m_{n}}, \ldots, L_{s_{n-1} m_{n}}\right] \cap L\left(L_{m_{n}}\right), L_{m_{n}}\right] \\
+\left(\operatorname{dim}\left[L_{m_{1}}, L_{m_{2}}, \ldots, L_{m_{n-1}}\right]-\operatorname{dim}\left(\left[L_{s_{1} m_{n}}, L_{s_{2} m_{n}}, \ldots, L_{s_{n-1} m_{n}}\right] \cap L\left(L_{m_{n}}\right)\right)\right) \operatorname{dim} L_{m_{n}} .
\end{array}
$$

By Lemma 3 (i), we have

$$
\left[L_{s_{1} m_{n}}, L_{s_{2} m_{n}}, \ldots, L_{s_{n-1} m_{n}}\right] \cap L\left(L_{m_{n}}\right)=\left[L_{s_{1}}\left(L_{m_{n}}\right), L_{s_{2}}\left(L_{m_{n}}\right), \ldots, L_{s_{n-1}}\left(L_{m_{n}}\right)\right] .
$$

Hence

$$
\begin{array}{r}
\operatorname{dim}\left[L_{m_{1}}, L_{m_{2}}, \ldots, L_{m_{n}}\right]=\operatorname{dim}\left[L_{s_{1}}\left(L_{m_{n}}\right), L_{s_{2}}\left(L_{m_{n}}\right), \ldots, L_{s_{n-1}}\left(L_{m_{n}}\right), L_{m_{n}}\right] \\
+\left(\operatorname{dim}\left[L_{m_{1}}, L_{m_{2}}, \ldots, L_{m_{n-1}}\right]-\operatorname{dim}\left[L_{s_{1}}\left(L_{m_{n}}\right), L_{s_{2}}\left(L_{m_{n}}\right), \ldots, L_{s_{n-1}}\left(L_{m_{n}}\right)\right]\right) \operatorname{dim} L_{m_{n}} .
\end{array}
$$

(iii) Clearly, $\left[L_{m_{1}}, L_{m_{2}}, \ldots, L_{m_{n}}\right]=\left[L_{m_{n}},\left[L_{m_{1}}, L_{m_{2}}, \ldots, L_{m_{n-1}}\right]\right]$. Then by applying Lemma 2 with $U=L_{m_{n}}$ and $V=\left[L_{m_{1}}, L_{m_{2}}, \ldots, L_{m_{n-1}}\right]$, we obtain

$$
\begin{array}{r}
\operatorname{dim}\left[L_{m_{1}}, L_{m_{2}}, \ldots, L_{m_{n}}\right]=\operatorname{dim}\left[L_{m_{n}},\left[L_{m_{1}}, L_{m_{2}}, \ldots, L_{m_{n-1}}\right]\right] \\
=\operatorname{dim}\left[L_{m_{n}} \cap L\left(\left[L_{m_{1}}, L_{m_{2}}, \ldots, L_{m_{n-1}}\right]\right),\left[L_{m_{1}}, L_{m_{2}}, \ldots, L_{m_{n-1}}\right]\right] \\
+\left(\operatorname{dim} L_{m_{n}}-\operatorname{dim}\left(L_{m_{n}} \cap L\left(\left[L_{m_{1}}, L_{m_{2}}, \ldots, L_{m_{n-1}}\right]\right)\right)\right) \operatorname{dim}\left[L_{m_{1}}, L_{m_{2}}, \ldots, L_{m_{n-1}}\right] .
\end{array}
$$

By our assumption, we have $L_{m_{n}} \cap L\left(\left[L_{m_{1}}, L_{m_{2}}, \ldots, L_{m_{n-1}}\right]\right)=0$. Therefore, 2 turns into the formula

$$
\operatorname{dim}\left[L_{m_{1}}, L_{m_{2}}, \ldots, L_{m_{n}}\right]=\operatorname{dim} L_{m_{n}} \operatorname{dim}\left[L_{m_{1}}, L_{m_{2}}, \ldots, L_{m_{n-1}}\right] .
$$


(iv) We use the same method as in (iii). We apply Lemma 2 with $U=L_{m_{n}}$ and $V=\left[L_{m_{1}}, L_{m_{2}}, \ldots, L_{m_{n-1}}\right]$, and since $m_{n}=s\left(m_{1}+m_{2}+\ldots+m_{n-1}\right)$, we have

$$
L_{m_{n}} \cap L\left(\left[L_{m_{1}}, L_{m_{2}}, \ldots, L_{m_{n-1}}\right]\right)=L_{s}\left(\left[L_{m_{1}}, L_{m_{2}}, \ldots, L_{m_{n-1}}\right]\right) .
$$

This implies that (2) turns into

$$
\begin{array}{r}
\operatorname{dim}\left[L_{m_{1}}, L_{m_{2}}, \ldots, L_{m_{n}}\right]=\operatorname{dim}\left[L_{s}\left(\left[L_{m_{1}}, L_{m_{2}}, \ldots, L_{m_{n-1}}\right]\right),\left[L_{m_{1}}, L_{m_{2}}, \ldots, L_{m_{n-1}}\right]\right] \\
+\left(\operatorname{dim} L_{m_{n}}-\operatorname{dim} L_{s}\left(\left[L_{m_{1}}, L_{m_{2}}, \ldots, L_{m_{n-1}}\right]\right)\right) \operatorname{dim}\left[L_{m_{1}}, L_{m_{2}}, \ldots, L_{m_{n-1}}\right] .
\end{array}
$$

Since $\left[L_{s}\left(\left[L_{m_{1}}, L_{m_{2}}, \ldots, L_{m_{n-1}}\right]\right),\left[L_{m_{1}}, L_{m_{2}}, \ldots, L_{m_{n-1}}\right]\right]=L_{s+1}\left(\left[L_{m_{1}}, L_{m_{2}}, \ldots, L_{m_{n-1}}\right]\right)$, we have the required result.

This main result gives formulae for the dimension of subspaces in the form $\left[L_{m_{1}}, L_{m_{2}}, \ldots, L_{m_{n}}\right]$ under certain conditions on $m_{1}, m_{2}, \ldots, m_{n}$. Unfortunately, the obstacle are the products in the forms $\left[L_{s_{1}}\left(L_{m_{n}}\right), L_{s_{2}}\left(L_{m_{n}}\right), \ldots, L_{s_{n-1}}\left(L_{m_{n}}\right), L_{m_{n}}\right]$ for $n \geq 3$.

Three immediate consequences of Theorem 4 are following.

Corollary 5 ([5], Theorem 1$)$. Let $m_{1}, m_{2}$ be positive integers with $m_{1} \geq m_{2}$.

(i) If $m_{1}>m_{2}$ and $m_{2} \nmid m_{1}$, then

$$
\operatorname{dim}\left[L_{m_{1}}, L_{m_{2}}\right]=\operatorname{dim} L_{m_{1}} \operatorname{dim} L_{m_{2}},
$$

(ii) and if $m_{1}=s m_{2}$ with $s \geq 1$, then

$$
\operatorname{dim}\left[L_{m_{1}}, L_{m_{2}}\right]=\left(\operatorname{dim} L_{m_{1}}-f\left(s, \operatorname{dim} L_{m_{2}}\right)\right) \operatorname{dim} L_{m_{2}}+f\left(s+1, \operatorname{dim} L_{m_{2}}\right) .
$$

Corollary 6 ([2], Theorem 3.1). Let $m_{1}, m_{2}$ and $m_{3}$ be positive integers with $m_{1} \geq$ $m_{2}$.

(i) If $m_{1}+m_{2}>m_{3}, m_{3} \nmid m_{1}$ or $m_{3} \nmid m_{2}$, then

$$
\operatorname{dim}\left[L_{m_{1}}, L_{m_{2}}, L_{m_{3}}\right]=\operatorname{dim}\left[L_{m_{1}}, L_{m_{2}}\right] \operatorname{dim} L_{m_{3}},
$$

(ii) if $m_{1}+m_{2}>m_{3}, m_{1}=s m_{3}$ and $m_{2}=t m_{3}$ with $s, t \geq 1$, then

$$
\begin{aligned}
\operatorname{dim}\left[L_{m_{1}}, L_{m_{2}}, L_{m_{3}}\right]= & \operatorname{dim}\left[L_{s}\left(L_{m_{3}}\right), L_{t}\left(L_{m_{3}}\right), L_{m_{3}}\right] \\
& +\left(\operatorname{dim}\left[L_{m_{1}}, L_{m_{2}}\right]-\operatorname{dim}\left[L_{s}\left(L_{m_{3}}\right), L_{t}\left(L_{m_{3}}\right)\right]\right) \operatorname{dim} L_{m_{3}},
\end{aligned}
$$

(iii) if $m_{3} \geq m_{1}+m_{2}$ and $\left(m_{1}+m_{2}\right) \nmid m_{3}$, then

$$
\operatorname{dim}\left[L_{m_{1}}, L_{m_{2}}, L_{m_{3}}\right]=\operatorname{dim}\left[L_{m_{1}}, L_{m_{2}}\right] \operatorname{dim} L_{m_{3}},
$$

(iv) and if $m_{3} \geq m_{1}+m_{2}$ and $m_{3}=s\left(m_{1}+m_{2}\right)$ with $s \geq 1$, then

$$
\begin{aligned}
\operatorname{dim}\left[L_{m_{1}}, L_{m_{2}}, L_{m_{3}}\right]= & \operatorname{dim} L_{s+1}\left(\left[L_{m_{1}}, L_{m_{2}}\right]\right) \\
& +\left(\operatorname{dim} L_{m_{3}}-\operatorname{dim} L_{s}\left(\left[L_{m_{1}}, L_{m_{2}}\right]\right)\right) \operatorname{dim}\left[L_{m_{1}}, L_{m_{2}}\right] .
\end{aligned}
$$




\section{REFERENCES}

[1] Magnus, W, Karrass, A and Solitar, D., Combinatorial Group Theory, Presentations of Groups in terms of Generators and Relations, New York, NY, USA Dover Publications, 2nd revised ed., 1976.

[2] Mansuroğlu, Ni and Stöhr, R., On the dimension of products of homogeneous subspaces in free Lie algebras, Internat. J. Algebra Comput., 1(23), (2013), 205-213.

[3] Shirshov, A.I., Subalgebras of free Lie algebras, Mat. Sb., 33:441-452, 1953 (in Russian).

[4] Shirshov, A.I., Selected works of A. I. Shirshov, Translated from the Russian by Murray Bremner and Mikhail V. Kotchetov, Edited by Leonid A. Bokut, Victor Latyshev, Ivan Shestakov and Efim Zelmanov, Contemporary Mathematicians, Birkhuser Verlag, Basel, viii+242 pp, 2009.

[5] Stöhr, R and Vaughan-Lee, M. Products of homogeneous subspaces in free Lie algebras, Internat. J. Algebra Comput., 5(19), (2009), 699-703.

[6] Witt, E., Treue Darstellungen Liescher Ringe, J. Reine Angew. Math., 177, (1937), 152-160.

[7] Witt, E., Die Unterringe der freien Lieschen Ringe, Math. Z., 64, (1956), 195-216.

Current address: Nil MANSUROĞLU: Department of Mathematics, Faculty of Arts and Science, Kırşehir Ahi Evran University, 40100, Kırşehir, Turkey

E-mail address: nil.mansuroglu@ahievran.edu.tr

ORCID Address: http://orcid.org/0000-0002-6400-2115 